\title{
Análisis de eficiencia multidireccional en hospitales públicos del Valle del Cauca*
}

\author{
Multidirectional Efficiency Analysis in Public Hospitals of Valle Del Cauca \\ Análise de eficiência multidirecional em hospitais públicos do Valle del Cauca
}

\author{
Kelly Patricia Murillo ${ }^{a}$ \\ Universidade de Aveiro, Portugal \\ ORCID: http://orcid.org/0000-0003-1479-8757 \\ Eugenio Alexandre Miguel Rocha \\ Universidade de Aveiro, Portugal \\ ORCID: http://orcid.org/0000-0003-3628-6795 \\ Carlos Alberto García González \\ Universidad Santiago de Cali, Colombia \\ ORCID: http://orcid.org/0000-0002-2944-9974
}

DOI: https://doi.org/10.11144/Javeriana.rgps17-34.aemh

Fecha de recepción: 30 Mayo 2017

Fecha de aceptación: 05 Octubre 2017

\section{Resumen:}

La crisis financiera de los hospitales públicos en Colombia conlleva un análisis detallado de cómo funcionan los diferentes servicios de salud, a fin de detectar fallas y generar una mejor utilización de los recursos. En esta investigación se estudiaron los niveles de eficiencia de 44 hospitales públicos del departamento del Valle del Cauca, referente a los servicios de ginecología y obstetricia, odontología y urgencias, entre los años 2007 y 2014 . Adoptamos un método determinístico no paramétrico reciente, hasta ahora no aplicado en estudios científicos de salud en Colombia, el cual combina el análisis de eficiencia multidireccional con otras relevantes técnicas para comparar grupos con diferentes niveles de eficiencia. Los resultados indicaron que más del $60 \%$ de los hospitales quedaron en el rango de poco eficientes, lo cual evidencia un inadecuado uso de los insumos (inputs). Esperamos motivar a burócratas a incorporar este tipo de técnicas de evaluación en su proceso de toma de decisiones.

Palabras clave: eficiencia, economía hospitalaria, evaluación en salud, gestión de recursos, hospitales públicos, servicios de salud.

\section{Abstract:}

Financial crises in the Colombian public hospitals call for a detailed analysis on how its different health services work, in order to detect the failures and attain a better use of the resources. This research examined the efficacy levels in 44 public hospitals throughout the Valle del Cauca province, covering the gynecology and obstetrics, dentistry, and emergency services between 2007 and 2014. A recent non-parametric deterministic method was used. Thus far, it has not been applied to health scientific studies in Colombia. It combines the multidirectional efficacy analysis with other relevant techniques in order to compare groups with different efficiency levels. Results showed that more than $60 \%$ of the hospitals were in the poor efficiency level, which evinces they are not making an appropriate use of their inputs. This report is expected to encourage the bureaucrats to include evaluation techniques like this in their decision-making processes.

Keywords: efficacy, hospital economy, health evaluation, resource management, public hospitals, health services.

\section{Resumo:}

A crise financeira dos hospitais públicos na Colômbia envolve uma análise detalhada de como funcionam os diferentes serviços de saúde, a fim de detectar falhas e gerar um melhor uso dos recursos. Nesta pesquisa estudaram-se os níveis de eficiência de 44 hospitais públicos do departamento do Valle del Cauca, referente aos serviços de ginecologia e obstetrícia, odontologia e urgências, entre os anos 2007 e 2014. Pegamos um método determinístico não paramétrico recente, até agora não aplicado em estudos científicos de saúde na Colômbia, o qual combina análise de eficiência multidirecional a outras relevantes técnicas para comparar grupos com diferentes níveis de eficiência. Os resultados indicaram que mais de 60\% dos hospitais ficou na faixa de pouco eficientes, o que evidencia um inadequado uso dos insumos (inputs). Esperamos motivar os burocratas para incorporarem este tipo de técnicas de avaliação no seu processo de tomada de decisões.

Palavras-chave: eficiência, economia hospitalar, avaliação em saúde, gestão de recursos, hospitais públicos, serviços de saúde.

\footnotetext{
${ }^{a}$ Autora de correspondencia. Correo electrónico: kelpamur@hotmail.com
} 


\section{Introducción}

Las nuevas teorías del crecimiento económico han planteado una relación directa entre la salud de la población, las mejoras en la productividad económica y el desarrollo de un país, por el beneficio social que de ella se deriva y por el papel que desempeña en la base del capital humano, convirtiendo a las políticas públicas de salud en temas de gran importancia y controversia mundial. Al considerar que el estado de salud de un individuo forma parte del capital humano acumulado y, por tanto, resulta clave para su capacidad de generar ingresos y riqueza en el mercado [1,2,3], se implementan nuevas políticas y se definen estrategias de control y reestructuración que permitan un avance significativo en este campo. En algunos países de América Latina estos cambios han respondido a reformas generales del Estado, como en Perú desde 1995; reformas de la Constitución como en Colombia y Ecuador; e igualmente reformas del proceso de modernización del Estado como en Argentina y Chile desde 1994 [4].

Específicamente en Colombia, en las dos últimas décadas, debido a la ineficiencia en la prestación de los servicios de salud se viene reformando el sistema, a través de normas ${ }^{1}$ que conllevan proporcionar una cobertura universal del aseguramiento, facilitar el acceso de las personas con menos recursos, mejorar la calidad y fomentar la eficiencia en la prestación de servicios por parte de los hospitales públicos. En tal sentido, es importante mantener una mirada crítica y generar una búsqueda constante de estrategias y técnicas que permitan evaluar de manera cada vez más detallada la eficiencia del sistema de salud. Esta evaluación debe considerar el sector sanitario en todas sus facetas y, desde luego, conlleva estudiar la eficiencia de los diferentes servicios hospitalarios ofrecidos a la comunidad.

El objetivo principal de esta investigación es estudiar el nivel de eficiencia sobre los servicios de salud: Ginecología y Obstetricia (GO); Odontología (O) y Urgencias (U), que son prestados en 44 hospitales públicos de nivel 1 de atención [5 ${ }^{2}$ del departamento del Valle del Cauca, a la población pobre asegurada y no asegurada, entre los años 2007 y 2014.

Para la presente investigación utilizamos un método determinístico no paramétrico reciente, basado en el análisis de eficiencia multidireccional (MEA, por sus siglas en inglés), propuesto por Bogetdet et al. [26], para medir la eficiencia relativa de unidades de decisión con múltiples entradas y múltiples salidas. MEA constituye una herramienta muy favorable e innovadora en el análisis de datos, desde que en este modelo la reducción de entradas/insumos y la expansión de salidas/productos se seleccionan proporcionalmente, buscando una mejora potencial por separado en cada variable de entrada y cada variable de salida (véase sección "Materiales y métodos"). En ese sentido, puede ser aplicado en muchos contextos, desde que facilita la estimación de la productividad específica de las variables usadas. Algunos ejemplos de la utilización de MEA en patrones de eficiencia en salud, que se puede examinar, son los trabajos de Hougaard et al. [7], en el cual la utilidad potencial de MEA como herramienta de gestión se ilustra utilizando un conjunto de datos de unidades de cirugía ortopédica danesas. En [8], por medio de MEA, se examina la utilización de los recursos de atención de ancianos daneses. Para aplicaciones recientes de MEA en otros contextos, invitamos al lector a consultar artículos como el de Asmild et al. [9] sobre análisis de eficiencia en bancos chinos entre 1997 y 2008, de Wang et al. [10] sobre la eficiencia regional de energía y emisiones de China entre 1997-2010, y de Asmild et al. [11] sobre eficiencia agrícola, el cual analiza tanto la eficiencia de gestión como la de programas de las explotaciones familiares lituanas, mostrando claras diferencias entre las puntuaciones de eficiencia en los diferentes insumos ${ }^{3}$.

Si bien es cierto que se han presentado avances en el análisis de la eficiencia hospitalaria en Colombia, en conocimiento de los autores esta constituye la primera vez que se usa el modelo MEA aplicado a la eficiencia de hospitales en el territorio nacional. En efecto, en Colombia, encontramos interesantes artículos sobre la eficiencia hospitalaria, aunque hasta el momento, no se encuentra en la literatura estudios que hayan utilizado MEA para medir el nivel de eficiencia sobre los servicios de salud. Con mayor frecuencia las investigaciones 
utilizan el análisis envolvente de datos (DEA, por sus siglas en inglés), a pesar de que el método MEA permite obtener una información más detallada sobre la ineficiencia de las variables usadas. La diferencia es principalmente porque usando MEA obtenemos un conjunto de puntuaciones de eficiencia específicas de entrada (o salida) para cada unidad estudiada (véase sección "Materiales y método").

El presente estudio pretende contribuir en dos aspectos a la literatura empírica sobre los determinantes de la eficiencia en hospitales públicos, en el contexto de países de ingresos medios altos con alta desigualdad en la distribución del ingreso y de la riqueza. En primer lugar, se realiza un enfoque integral del análisis de eficiencia, al poner bajo análisis a tres servicios de salud dentro de los hospitales: GO, O y U. En segundo lugar, el uso del modelo MEA como instrumento completo e innovador de análisis no antes usado para medir eficiencia en el sector salud que permite explotar la riqueza de la base de datos empleada.

Por otro lado, con este análisis pretendemos contribuir a los mandatarios, autoridades ejecutivas locales, burócratas y gestores de salud en la toma de decisiones de política pública y de gestión pública, de tal manera que puedan mejorar en un mediano y largo plazo, no solamente la situación financiera, sino también la eficiencia y la calidad de los servicios de salud que son prestados a la población pobre asegurada y pobre no asegurada que acude a los hospitales públicos de la red del Valle del Cauca.

\section{Materiales y método}

Evaluar la calidad del sistema de salud conlleva estudiar la eficiencia de los diferentes servicios hospitalarios ofrecidos a la comunidad. Según Giedion et al. [12], la eficiencia de un hospital depende de dos aspectos principales. El primero, relacionado con el uso de los insumos, se conoce como eficiencia técnica, que relaciona las variables inputs/insumos utilizadss por el hospital, debido a los outputs/productos que se generan. El segundo aspecto, relacionado con los costos de los factores productivos (inputs/insumos), se denomina eficiencia económica e incluye consideraciones de minimización de dichos costos. En la literatura económica, la medición de la eficiencia de las organizaciones se puede llevar a cabo mediante el análisis de frontera paramétrica o no paramétrica. En el caso de las organizaciones de salud, los dos métodos más utilizados son el enfoque de análisis de regresión y el DEA, los cuales requieren en su desarrollo los métodos econométricos y de programación lineal, respectivamente [13].

\section{DEA versus MEA}

El método DEA ha sido utilizado en muchos contextos, desde que permite resolver problemas de maximización simultánea de productos o minimización simultánea de insumos, construyendo una frontera de producción óptima y comparando cada unidad de observación con el óptimo esperado. Sin embargo, debido a que el modelo DEA se basa en las contracciones radiales en todas las entradas y salidas indeseables, y en expansiones radiales en todas las salidas deseables, no es el más adecuado para evaluar los patrones de la eficiencia en los hospitales y establecer índices de ineficiencia en cada variable individualmente. Lo anterior es posible hacerlo usando el método determinístico no paramétrico reciente MEA, en el cual la reducción de entradas y la expansión de salidas se seleccionan proporcionalmente, buscando una mejora potencial por separado en cada variable de entrada y cada variable de salida. En la investigación de Hougaard et al. [8], por ejemplo, se demuestra que MEA proporciona una imagen de rendimiento mucho más sutil que DEA, porque con ese modelo se consigue evaluar los potenciales de mejora relativa específicas de los insumos. En [14] se demuestra que al usar MEA en el factor total del índice Malmquist - índice multilateral usado para comparar la productividad (tecnología de producción y eficiencia) de diversas economías - sobre granjas familiares de Lituania, en vez del enfoque convencional DEA, es posible obtener un análisis específico de variables, cambios en la productividad, así como sus componentes. Los resultados destacan que se puede ocultar diferencias 
importantes en el rendimiento específico de las granjas cuando se utiliza el convencional (radial) DEA basado en el índice Malmquist.

Para otros ejemplos de artículos científicos en otros contextos, donde son claramente detectados los beneficios de MEA, el lector puede revisar el trabajo de Asmild et al. [15], en el cual el uso de MEA permite investigar cómo las reformas ferroviarias afectan las ineficiencias de los factores de costo específicos. En [16], MEA permite no solo evaluar el nivel de eficiencia energética y ambiental y la tendencia del sector de transporte de China, sino también investigar los patrones de eficiencia de treinta regiones y tres áreas principales de China, durante el periodo 2006-2010. En este mismo país, Wang et al. [17] detectaron tanto los niveles de eficiencia MEA integrados como los patrones de eficiencia, los cuales están representados por la eficiencia MEA específica de la variable según cada tipo de emisión o descarga de contaminantes industriales de la ciudad principal. En esta investigación también se miden los potenciales de conservación de energía industrial y de reducción de contaminantes. Además, se explora la relación entre la presión ambiental y los ingresos a nivel regional de China.

A continuación, presentamos una síntesis de algunos de los estudios nacionales consultados que evaluaron la eficiencia en grupos de hospitales públicos y privados: Rojas [18] determinó los niveles de eficiencia de hospitales públicos de nivel II localizadas en el departamento del Tolima, mediante la técnica DEA, en el periodo 2002-2005. Mora et al. [19] evaluaron el producto total hospitalario y el recurso humano de 404 instituciones proveedoras de servicios de salud hospitalarios, mediante relaciones de equivalencia. Giedion et al. [12] evaluaron la eficiencia económica y de gestión de los 31 hospitales públicos de Bogotá entre 1994 y 1997, mediante una correlación entre costo del producto hospitalario y gasto. Polanía [20] aplica la técnica DEA para analizar la eficiencia de 143 hospitales públicos y privados del país en 1996. Nupia et al. [21] abordan la eficiencia de los 31 hospitales públicos de Bogotá, utilizando información de producción e insumos de los hospitales en 1999.

Mediante DEA, se obtienen medidas de eficiencia, tanto técnicas como asignativas, usando funciones de producción multiproducto y uniproducto, respectivamente, con diferentes supuestos de rendimientos a escala. Pinzón [22], en una primera etapa, recolectó información de 203 hospitales públicos colombianos de baja complejidad en el año 2001, y estimó los coeficientes de eficiencia técnica relativa en los hospitales mediante DEA. En la segunda etapa estimó un modelo Tobit o modelo de regresión censurada, así como un modelo de mínimos cuadrados ordinarios. En [23] la eficiencia se mide desde dos perspectivas, la primera, dada por los indicadores de recursos, aprovechamiento, funcionamiento y calidad, y la segunda, dada por un modelo de frontera estocástica para medir la eficiencia de 616 hospitales públicos nacionales. Toro et al. [24], mediante el uso de DEA, evidenciaron que la ineficiencia presente en un alto porcentaje de hospitales públicos en Colombia estaba asociada a la baja complejidad de sus servicios. Fontalvo et al. [25] estudiaron la eficiencia de 44 hospitales de Bolívar en el año 2010, utilizando el modelo DEA enfocado en salidas, por medio del estudio de los estados financieros.

A continuación presentamos una descripción general del modelo MEA.

\section{Modelo MEA}

Sea I; J; T $\epsilon$ N y defina $[\mathrm{m}]=\{1, \ldots, \mathrm{m}\}$. Considere una terna $\mathrm{n}=(\mathrm{h}, \mathrm{s}, \mathrm{t}) \epsilon \mathrm{HxSxN}$, identificando un hospital h $\ddot{\epsilon}$ $\mathrm{H}$, un servicio de salud $s \in S$ y un año $t \in T$. Suponga que cualquier terna $n \in N$ produce $y_{j}(n), j \in[J]$ salidas/ ouputs, usando $\mathrm{x}_{\mathrm{i}}(\mathrm{n}), \mathrm{i} \in[\mathrm{I}]$ entradas/inputs, donde las primeras entradas $1<\mathrm{D} \leq \mathrm{I}$ son las llamadas entradas discrecionales (variables de entrada que intervienen en el proceso de optimización). Por tanto, $\mathrm{x}(\mathrm{n}) \in \mathrm{R}^{\mathrm{I}}$ es el vector de todas las entradas, $\mathrm{y}(\mathrm{n}) \in \mathrm{R}^{\mathrm{J}}$ es el vector de todas las salidas, y el conjunto de valores $\mathrm{z}(\mathrm{n})=(\mathrm{x}(\mathrm{n}) ; \mathrm{y}(\mathrm{n}))$ es la base de datos para cada terna hospital/servicio/año $\mathrm{n}$ dada. 
Considere el conjunto

$$
\Lambda^{n}=\left\{\lambda \in \mathrm{R}^{N}: \sum_{n=1}^{N} \lambda_{n}=1 \wedge \lambda n \geq 0\right\}
$$

El modelo MEA para una observación especifica $E_{0}$ es calculado resolviendo los siguientes programas de optimización lineal.

$$
\begin{array}{ll}
P_{m}{ }^{\alpha}(z, \bar{n}): & P \beta(z, \bar{n}): \\
\min \alpha_{\mathrm{m}}(\overline{\mathrm{n}}) \text { tal que } & \max \beta_{\mathrm{j}}(\overline{\mathrm{n}}) \text { tal que } \\
\sum_{\mathrm{n}} \lambda_{\mathrm{n}} \mathrm{x}_{\mathrm{m}}(\mathrm{n}) \leq \alpha_{\mathrm{m}}(\overline{\mathrm{n}}) & \sum_{\mathrm{n}} \lambda_{\mathrm{n}} \mathrm{x}_{\mathrm{i}}(\mathrm{n}) \leq \mathrm{x}_{\mathrm{i}}(\overline{\mathrm{n}}), \mathrm{i} \in[\mathrm{I}] \\
\sum_{\mathrm{n}} \lambda_{\mathrm{n}} \mathrm{x}_{\mathrm{i}}(\mathrm{n}) \leq \mathrm{x}_{\mathrm{i}}(\overline{\mathrm{n}}), \mathrm{i} \in[\mathrm{I}], \mathrm{i} \neq \mathrm{m} & \sum_{\mathrm{n}} \lambda_{\mathrm{n}} \mathrm{y}_{\mathrm{j}}(\mathrm{n}) \leq \beta_{\mathrm{j}}(\overline{\mathrm{n}}) \\
\sum_{\mathrm{n}} \lambda_{\mathrm{n}} \mathrm{y}_{\mathrm{l}}(\mathrm{n}) \leq \mathrm{y}_{\mathrm{l}}(\overline{\mathrm{n}}), 1 \in[\mathrm{J}] & \\
& \sum_{\mathrm{n}} \lambda_{\mathrm{n}} \mathrm{y}_{\mathrm{l}}(\mathrm{n}) \leq \mathrm{y}_{\mathrm{l}}(\overline{\mathrm{n}}), 1 \in[\mathrm{J}], \mathrm{l} \neq \mathrm{j} \\
P^{\gamma}(\alpha, \beta, z, n): & \\
\max \gamma(\overline{\mathrm{n}}) \text { tal que } & \\
\sum_{\mathrm{n}} \lambda_{\mathrm{n}} \mathrm{x}_{\mathrm{i}}(\mathrm{n}) \leq \mathrm{x}_{\mathrm{i}}(\overline{\mathrm{n}})-\gamma(\overline{\mathrm{n}})\left(\mathrm{x}_{\mathrm{i}}(\overline{\mathrm{n}})-\alpha_{\mathrm{i}}{ }^{*}(\overline{\mathrm{n}})\right), \mathrm{i} \in[\mathrm{M}] & \\
\sum_{\mathrm{n}} \lambda_{\mathrm{n}_{\mathrm{n}}} \mathrm{x}_{\mathrm{i}}(\mathrm{n}) \leq \mathrm{x}_{\mathrm{i}}(\overline{\mathrm{n}}), \mathrm{i} \in[\mathrm{I}] \backslash\{\mathrm{m}\} & \\
\sum_{\mathrm{n}} \lambda_{\mathrm{n}} \mathrm{y}_{\mathrm{l}}(\mathrm{n}) \geq \mathrm{y}_{\mathrm{l}}(\overline{\mathrm{n}})+\gamma(\overline{\mathrm{n}})\left(\beta_{1} *(\overline{\mathrm{n}})-\mathrm{y}_{\mathrm{l}}(\overline{\mathrm{n}})\right), 1 \in[\mathrm{J}] &
\end{array}
$$

donde $\mathrm{MEA}_{z}(\mathrm{n})$ son las soluciones óptimas para los problemas $\mathrm{R}_{i}(\mathrm{n})$, respectivamente. De esta manera, el punto de referencia ideal de $E_{0}$ es dado por el siguiente vector de salida MEA,

$$
\zeta(\overline{\mathrm{n}})=\left(\alpha_{1}{ }^{*}(\overline{\mathrm{n}}), \ldots, \alpha_{\mathrm{d}}^{*}(\overline{\mathrm{n}}), \mathrm{x}_{\mathrm{d}+1}(\overline{\mathrm{n}}), \ldots, \mathrm{x}_{\mathrm{l}}(\overline{\mathrm{n}}), \beta_{1}^{*}(\overline{\mathrm{n}}), \ldots, \beta_{\mathrm{J}}^{*}(\overline{\mathrm{n}})\right)
$$

En este sentido, el modelo MEA para una específica observación $E_{1}$ consiste de un total de $E_{0} \mathrm{x} \mathrm{N}$ programas lineales, lo cual incluye un problema $E_{1}$ para cada variable discrecional d $\epsilon \mathrm{D}$, un problema $E_{1}$ para cada una de las salidas $\mathrm{j} \epsilon[\mathrm{J}]$ y un problema $E_{0}$ con las soluciones optimas de los problemas $E_{0}$.

En otras palabras, el modelo MEA, consiste en encontrar:

$$
\begin{aligned}
& \alpha_{\mathrm{m}}^{*}(\overline{\mathrm{n}}) \in \mathrm{R}^{\mathrm{D}}, \text { tal que } \alpha_{\mathrm{m}} *(\overline{\mathrm{n}}) \in P_{m}^{\alpha}(z, \bar{n}) ; \\
& \left.\beta_{\mathrm{J}}^{*}(\overline{\mathrm{n}}) \in \mathrm{R}^{\mathrm{J}}, \text { tal que } \beta_{\mathrm{j}}^{*}(\overline{\mathrm{n}})\right) \in P_{j}^{\beta}(z, \bar{n}) ; \\
& \gamma(\overline{\mathrm{n}}) \in \mathrm{R}, \text { tal que } \gamma \in P^{\gamma}(\alpha, \beta, z, \bar{n}) .
\end{aligned}
$$

Para un conjunto de datos $z=\{z(n)\}_{N}$, con $z(n)=(x(n) ; y(n))$ el coeficiente $M E A$ se define por medio de la siguiente ecuación: 


$$
\operatorname{MEA}_{z}(\mathrm{n})=\frac{\frac{1}{\gamma(\overline{\mathrm{n}})}-\frac{1}{D} \sum_{i=1}^{D} \frac{x_{i}(\overline{\mathrm{n}})-a_{i}^{*}(\overline{\mathrm{n}})}{x_{i}(\overline{\mathrm{n}})}}{\frac{1}{\gamma(\overline{\mathrm{n}})}+\frac{1}{J} \sum_{j=1}^{J} \frac{\beta_{j}^{*}(\overline{\mathrm{n}})-y_{j}(\overline{\mathrm{n}})}{y_{j}(\overline{\mathrm{n}})}}
$$

donde $\alpha_{i}{ }^{*}(\bar{n})$ y $\beta_{j}{ }^{*}(\bar{n})$ representan las soluciones optimas correspondientes a los problemas de optimización lineal $P_{m}{ }^{\alpha}(z, \bar{n})$ y $P_{m}{ }^{\beta}(z, \bar{n})$, respectivamente.

El coeficiente MEA se obtiene por medio de la contribución direccional de cada entrada y salida. En efecto, en las entradas, la contribución para cada unidad $z(\bar{n})$ es dada por:

$$
\operatorname{meff}_{i}(\bar{n})=\frac{x_{i}(\overline{\mathrm{n}})-\gamma(\overline{\mathrm{n}})\left(\mathrm{xi}(\overline{\mathrm{n}})-a_{i}^{*}(\overline{\mathrm{n}})\right)}{x_{i}(\overline{\mathrm{n}})} \chi_{D}(i)
$$

para $\mathrm{i} \in[\mathrm{I}]$ donde $\chi_{D}(i)$ representa la función característica del conjunto D.

En las salidas, la contribución para cada unidad $z(\bar{n})$ es dada por ${ }_{j} \in[\mathrm{J}]$ por

$$
\operatorname{meff}_{j}(\overline{\mathrm{n}})=\frac{y_{j}(\overline{\mathrm{n}})}{y_{i}(\overline{\mathrm{n}})+\gamma(\overline{\mathrm{n}})\left(\beta_{i}{ }^{*}(\overline{\mathrm{n}})-\mathrm{yl}(\overline{\mathrm{n}})\right)} .
$$

Nótese que $\gamma(\overline{\mathrm{n}})\left(\mathrm{x}_{\mathrm{i}}(\overline{\mathrm{n}})-a_{i}^{*}(\overline{\mathrm{n}})\right)$ representa el exceso de entrada para todo $\mathrm{i}=1, \ldots, \mathrm{D}$, e $\gamma(\overline{\mathrm{n}})\left(\beta_{1}{ }^{*}(\overline{\mathrm{n}})-\mathrm{y}_{1}(\overline{\mathrm{n}})\right)$ representa la insuficiencia de salida para todo $\mathrm{j}=1, \ldots . \mathrm{J}$.

Una característica muy interesante del modelo MEA es que la ineficiencia de cada variable de entrada, puede ser analizada individualmente. En efecto, con base en el exceso de entrada e usando las ideas en Bogetoft et al. [6], definimos el siguiente indice de ineficiencia MEA:

$$
\mathrm{R}_{i}(\mathrm{n})=\frac{\sum_{n=1}^{N} \gamma(\mathrm{n})\left(x_{i}(\mathrm{n})-a_{i}^{*}(\mathrm{n})\right)}{\sum_{n=1}^{N} x_{i}(\mathrm{n})}
$$

para todo i $\epsilon[\mathrm{I}]$ y terna $\mathrm{n} \epsilon \mathrm{N}$. Referimos el índice de ineficiencia para saber el número de veces en que cada entrada fue usada ineficientemente.

\section{Cálculo del valor-NC}

Analizar el comportamiento de grupos con diferentes niveles de eficiencia es un aspecto de gran importancia en el análisis de datos. Las estadísticas del grupo eficiente con las del grupo no eficiente en este artículo 
son comparadas utilizando una técnica desarrollada por Inman et al. [27], que se basa en un coeficiente de intersección de distribución. Llamamos a este coeficiente valor-NC, para referirnos a las normales de distribución cruzadas. La idea es la siguiente: consideramos dos grupos para cada servicio de salud, de acuerdo con el coeficiente MEA:

- $\operatorname{MEA}_{z}(\mathrm{n})$ : grupo correspondiente a los hospitales más eficientes, definido como las unidades con coeficiente MEA $\geq 0.6$;

- $\mathrm{R}_{i}(\mathrm{n})$ : grupo correspondiente a los hospitales menos eficientes, definido como las unidades con coeficiente MEA $\leq 0.4$.

El valor-NC se calcula como la intersección de las funciones gaussianas asociadas a las eficiencias mostradas en $E_{1}$ y $E_{0}$. Cuanto mayor sea el valor de $\mathrm{NC}$, menos común será el comportamiento de los dos grupos con respecto a las variables seleccionadas. De esta manera, es posible comparar el comportamiento de las variables de entrada y salida entre grupos con diferentes niveles de eficiencia y determinar desde ese punto de vista su influencia en el modelo MEA.

\section{Datos y variables}

En este artículo se estudia la eficiencia de los servicios: Ginecología y Obstetricia (GO), Odontología (O) y Urgencia (U), en 44 de los $45^{4}$ hospitales públicos existentes de nivel I de atención, concentrados en 39 de los $42^{5}$ municipios del departamento del Valle del Cauca. Para esto, utilizamos como fuente de datos el Sistema de Información Hospitalario del Ministerio de Salud y Protección Social (SIHO). Con el fin de tener una perspectiva de evolución sobre el tiempo, para un análisis de la eficiencia técnica más detallada, se han utilizado datos desde el año 2007 hasta el 2014.

TABLA 1

\section{VARIABLES EN CADA SERVICIO DE SALUD}

\begin{tabular}{|c|c|}
\hline \multicolumn{2}{|c|}{ Servicio de Gine cología y Obste tricia (GO) } \\
\hline Variables de entrada/inputs & Variables de salida/outputs \\
\hline $\begin{array}{l}\text { [V 205]: Citologias cervicovaginales tomadas; } \\
\text { [V 253]: Camas de hospitalización; } \\
\text { [V 258]: Camas de parto. }\end{array}$ & $\begin{array}{l}\text { [V 220]: Salidas obstetricias*; } \\
\text { [V 445]: Pacientes remitidos a niveles superiores. }\end{array}$ \\
\hline \multicolumn{2}{|c|}{ Servicio de Odontología $(O)$} \\
\hline Variables de entrada/inputs & Variables de salida/outputs \\
\hline $\begin{array}{l}\text { [V 211]: Consultas odontológicas realizadas; } \\
\text { [V 259]: Unidades odontológicas; } \\
\text { [V 681]: Consultas odontológicas asignadas. }\end{array}$ & $\begin{array}{l}\text { [V 212]: Sellantes aplicadas; } \\
\text { [V 213]: Superficies selladas (cualquier material); } \\
\text { [V 214]: Exodoncias (cualquier tipo). }\end{array}$ \\
\hline \multicolumn{2}{|c|}{ Servicio de Urgencias (U) } \\
\hline Variables de entrada/inputs & Variables de salida/outputs \\
\hline $\begin{array}{l}\text { [V 208]: Consultas médicas urgentes realizadas; } \\
\text { [V 254]: Camas de observación; } \\
\text { [V 256]: Consultorios; } \\
\text { [V 257]: Camas de cirugia; } \\
\text { [V 679]: Pacientes atendidos. }\end{array}$ & $\begin{array}{l}\text { [V 240]: Exámenes de laboratorio; } \\
\text { [V 241]: Imágenes de diagnóstico tomadas; } \\
\text { [V 444]: Pacientes remitidos a niveles superiores. } \\
\text { [V 684]: Pacientes hospitalizados. }\end{array}$ \\
\hline
\end{tabular}


Para cada tipo de servicio hemos extraído las variables de entrada/inputs y salida/outputs que se presentan en la tabla 1. Los números que acompañan a la letra $\mathrm{V}$ (variables) corresponden a la codificación en la base de datos SIHO del Ministerio de Salud y Protección Social de la República de Colombia. Las estadísticas (suma, media y desviación estándar) de todas las variables utilizadas en este estudio se proporcionan en las tablas 5, 6 y 7. Al igual que otros investigadores, estamos sujetos a trabajar con una base estructurada que contiene bastantes datos confiables. En nuestro caso, al momento de armonizar los registros por servicios de salud: $\mathrm{GO}, \mathrm{O}$ y $\mathrm{U}$, utilizamos todas las variables disponibles y completas para cada uno de los años de estudio, periodo comprendido entre 2007 y 2014.

\section{Resultados}

Nuestros resultados involucran las relaciones de eficiencia en cada uno de los tres servicios de salud considerados, el índice de ineficiencia de los insumos y los resultados del valor-NC entre los hospitales más y menos eficientes.

\section{Relaciones de eficiencia}

Los resultados obtenidos al aplicar el modelo MEA a los datos suministrados por SIHO (tablas 5, 6 y 7 , ubicadas al final del texto) se clasifican y se presentan de la siguiente forma: una vez obtenido el coeficiente de eficiencia $\mathrm{MEA}_{z}(\mathrm{n})$ en cada uno de los tres servicios de salud, en cada año y cada hospital, definimos el conjunto de las unidades eficientes EFF como

$$
E F F=\left\{n: 0.6 \leq \operatorname{MEA}_{z}(\mathrm{n}) \leq 1\right\}
$$

y obtenemos la eficiencia media total y la ratio EFF/Total. Este último es calculado como el coeficiente entre el número de unidades eficientes y el número total de unidades. Los resultados de estas dos medidas en los tres servicios se presentan en la tabla 2. El número entre paréntesis en la columna de EFF/Total corresponde al número de unidades que representa cada porcentaje. En la figura 1 se representa el comportamiento de esta ratio en cada servicio, a lo largo del tiempo, lo cual muestra progresivamente los cambios correspondientes a los niveles de eficiencia de cada servicio, evidenciando un comportamiento totalmente variable durante todo el periodo de estudio. Lo anterior implica una desajustada y poco favorable estrategia seguida por GO, O y $\mathrm{U}$, la mayor parte del tiempo. 
TABLA 2

Ratios de eficiencia

\begin{tabular}{|c|c|c|c|c|c|c|}
\hline \multirow{2}{*}{ AÑo } & \multicolumn{2}{|c|}{ SERVICIO GO } & \multicolumn{2}{|c|}{ SERVICIO O } & \multicolumn{2}{|c|}{ SERVICIOU } \\
\hline & Media Total & \begin{tabular}{|l|} 
EFF/Total \\
\end{tabular} & Media Total & EFF/Total & Media Total & EFF/Total \\
\hline 2007 & 0.36 & $11.4 \%(5$ in 44$)$ & 0.27 & $13.6 \%(6$ in 44$)$ & 0.61 & $45.5 \%(20$ in 44$)$ \\
\hline 2008 & 0.28 & $11.4 \%(5$ in 44$)$ & 0.36 & $15.9 \%$ (7 in 44$)$ & 0.62 & $40.9 \%$ (18 in 44$)$ \\
\hline 2009 & 0.23 & $9.09 \%$ (4 in 44$)$ & 0.38 & $13.6 \%(6$ in 44$)$ & 0.62 & $45.5 \%(20$ in 44$)$ \\
\hline 2010 & 0.52 & $27.3 \%$ (12 in 44$)$ & 0.33 & $15.9 \%$ (7 in 44$)$ & 0.54 & $34.1 \%$ (15 in 44$)$ \\
\hline 2011 & 0.45 & $22.7 \%(10$ in 44$)$ & 0.42 & $29.5 \%(13$ in 44$)$ & 0.68 & $52.3 \%(23$ in 44$)$ \\
\hline 2012 & 0.43 & $15.9 \%(7$ in 44$)$ & 0.51 & $31.8 \%(14$ in 44$)$ & 0.62 & $40.9 \%(18$ in 44$)$ \\
\hline 2013 & 0.3 & $13.6 \%(6$ in 44$)$ & 0.5 & $27.3 \%(12$ in 44$)$ & 0.72 & $54.5 \%(24$ in 44$)$ \\
\hline 2014 & 0.35 & $15.9 \%(7$ in 44$)$ & 0.45 & $27.3 \%(12$ in 44$)$ & 0.73 & $54.5 \%$ (24 in 44$)$ \\
\hline
\end{tabular}

Fuente: elaboración propia.

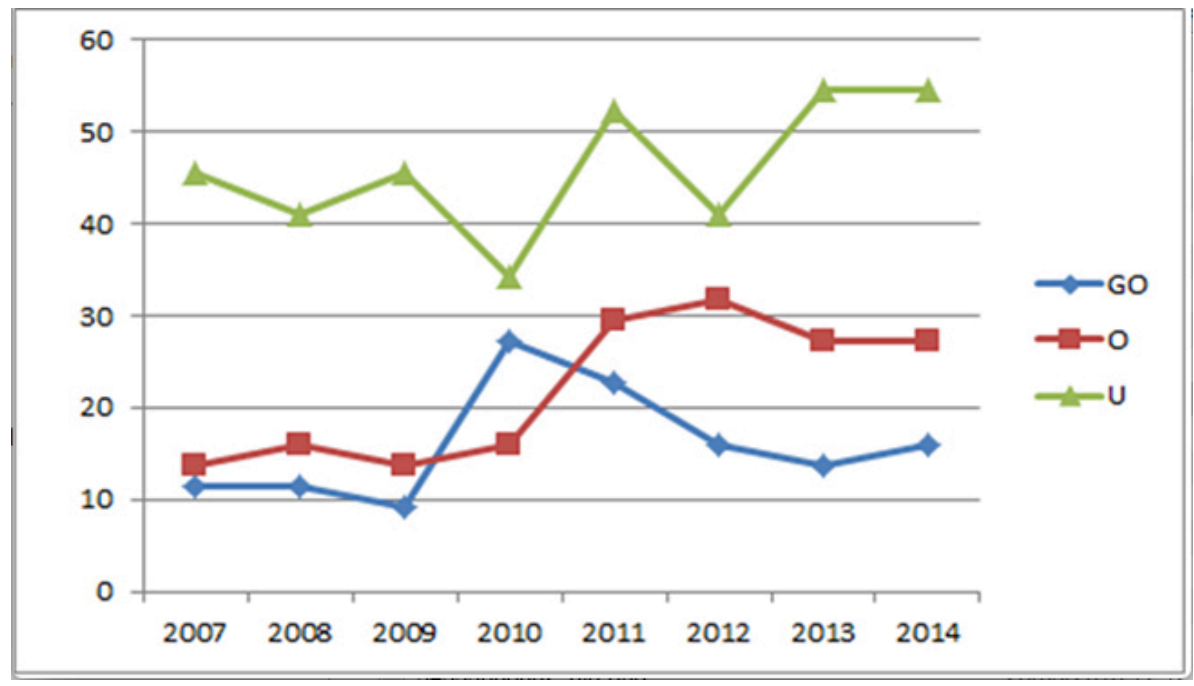

FIGURA 1

Eficiencia hospitalaria

Fuente: elaboración propia

\section{Indicadores de ineficiencia}

Para analizar con más detalle la contribución de cada variable en el modelo, se calculó el índice de ineficiencia $\mathrm{R}_{i}$ (n)para cada una de las variables de los tres servicios de salud, en cada año. Los resultados en la tabla 3 representan el número de veces que cada entrada se utilizó de manera ineficiente (exceso de insumos). En las figuras 2, 3 y 4 podemos ver la ineficiencia de cada insumo en los servicios de salud GO, O y U, respectivamente. 
TABLA 3

Índice de ineficencia

\begin{tabular}{|c|c|c|c|c|c|c|c|c|c|c|c|}
\hline \multirow{2}{*}{ AŇ̀o } & \multicolumn{3}{|c|}{ SERIICIO GO } & \multicolumn{3}{|c|}{ SERIICIO O } & \multicolumn{5}{|c|}{ SERIICIOU U } \\
\hline & V205 & $\mathrm{V} 253$ & V258 & V211 & V259 & v681 & V208 & $\mathrm{V} 254$ & $\mathrm{~V} 256$ & V257 & V679 \\
\hline 2007 & 0.26 & 0.47 & 0.45 & 0.49 & 0.48 & 0.54 & 0.14 & 0.31 & 0.24 & 0.34 & 0.15 \\
\hline 2008 & 0.40 & 0.52 & 0.45 & 0.44 & 0.51 & 0.52 & 0.15 & 0.24 & 0.22 & 0.31 & 0.21 \\
\hline 2009 & 0.48 & 0.55 & 0.75 & 0.42 & 0.52 & 0.50 & 0.15 & 0.31 & 0.24 & 0.21 & 0.20 \\
\hline 2010 & 0.13 & 0.31 & 0.34 & 0.41 & 0.47 & 0.55 & 0.22 & 0.38 & 0.30 & 0.33 & 0.24 \\
\hline 2011 & 0.22 & 0.38 & 0.42 & 0.31 & 0.32 & 0.35 & 0.12 & 0.20 & 0.18 & 0.11 & 0.16 \\
\hline 2012 & 0.22 & 0.35 & 0.41 & 0.21 & 0.29 & 0.25 & 0.14 & 0.25 & 0.16 & 0.39 & 0.15 \\
\hline 2013 & 0.36 & 0.53 & 0.52 & 0.19 & 0.25 & 0.26 & 0.09 & 0.19 & 0.12 & 0.21 & 0.12 \\
\hline 2014 & 0.40 & 0,41 & 0.54 & 0.20 & 0.28 & 0.25 & 0.08 & 0.21 & 0.11 & 0.23 & 0.08 \\
\hline
\end{tabular}

Fuente: elaboración propia.

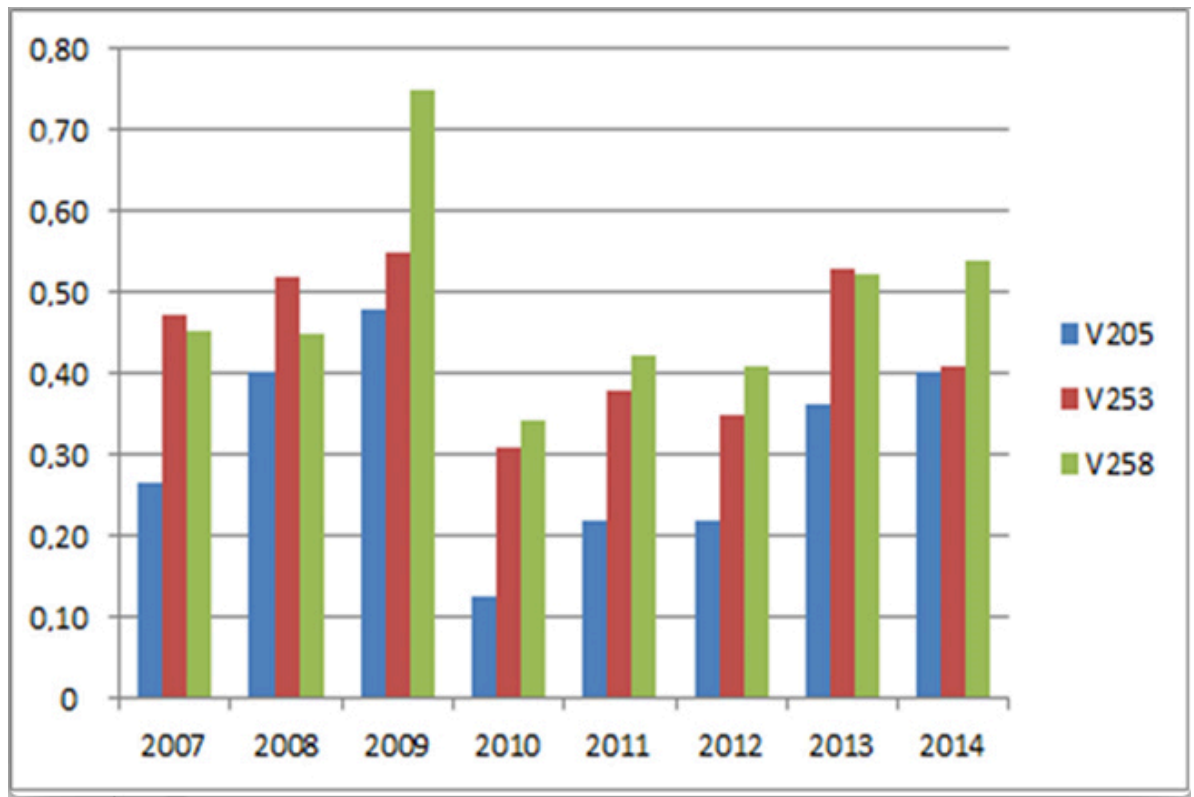

FIGURA 2

Indicador de ineficiencia del servicio de salud GO

Fuente: elaboración propia. 


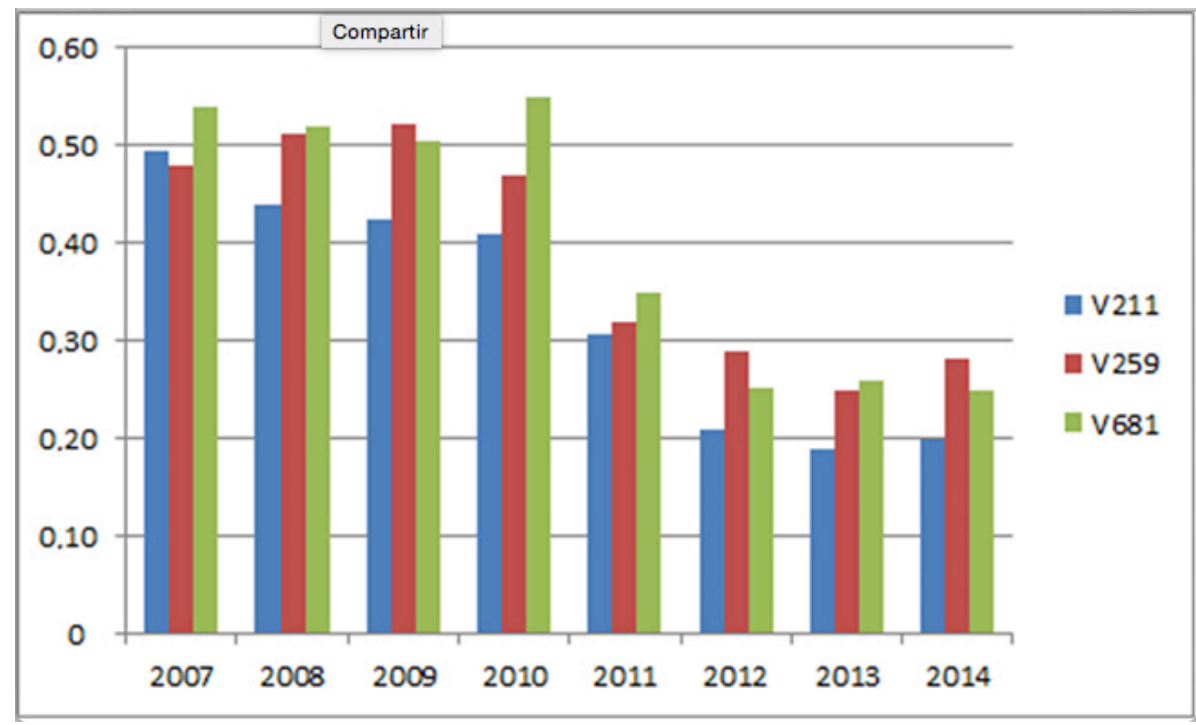

FIGURA 3

Indicador de ineficiencia del servicio de salud $\mathrm{O}$

Fuente: elaboración propia.

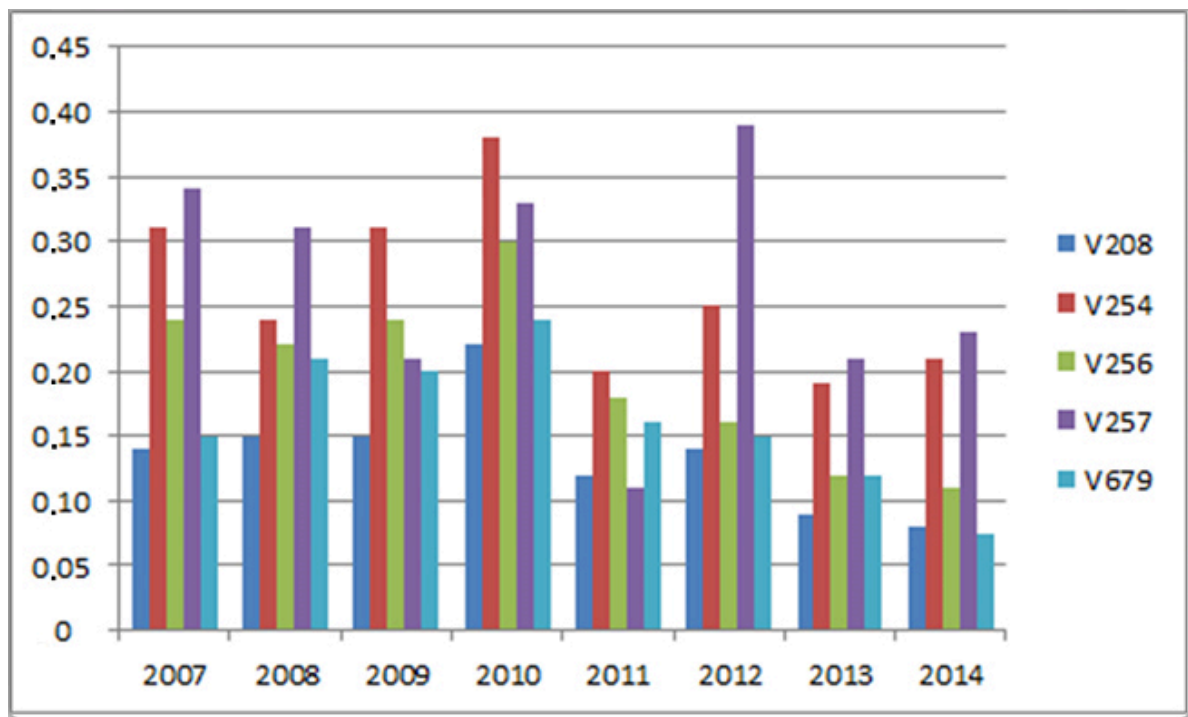

FIGURA 4

Indicador de ineficiencia del servicio de salud U Fuente: elaboración propia.

\section{Cálculo del valor-NC}

Después de obtener los resultados del coeficiente MEA de cada servicio, formamos el grupo de los hospitales menos eficientes $E_{0}$ y el grupo de los hospitales más eficientes $E_{1}$. Calculamos el valor NC para cada variable como la intersección de las funciones gaussianas asociadas a las eficiencias en cada grupo. En cada servicio de salud obtuvimos un gráfico detallado del comportamiento de los grupos eficientes y no eficientes, en cada variable. Es decir, obtuvimos un total de veinte gráficos, de acuerdo con el número de variables de cada servicio: cinco para el servicio de GO; seis para el servicio de $\mathrm{O}$ y nueve para el servicio de U. A continuación, describimos en detalle uno de estos gráficos. La figura 5 representa el comportamiento de los grupos de eficiencia $E_{0}$ y $E_{1}$ para la variable V684 (pacientes hospitalizados) en el servicio U. La media del grupo de los 
hospitales más eficientes la representa la línea azul, en tanto que la media del grupo de los hospitales menos eficientes la representa la línea negra. Las líneas roja y verde representan las desviaciones estándar para cada grupo. En este caso, $E_{1}=34, E_{0}=11$ y el valor- $\mathrm{NC}=416.9$.

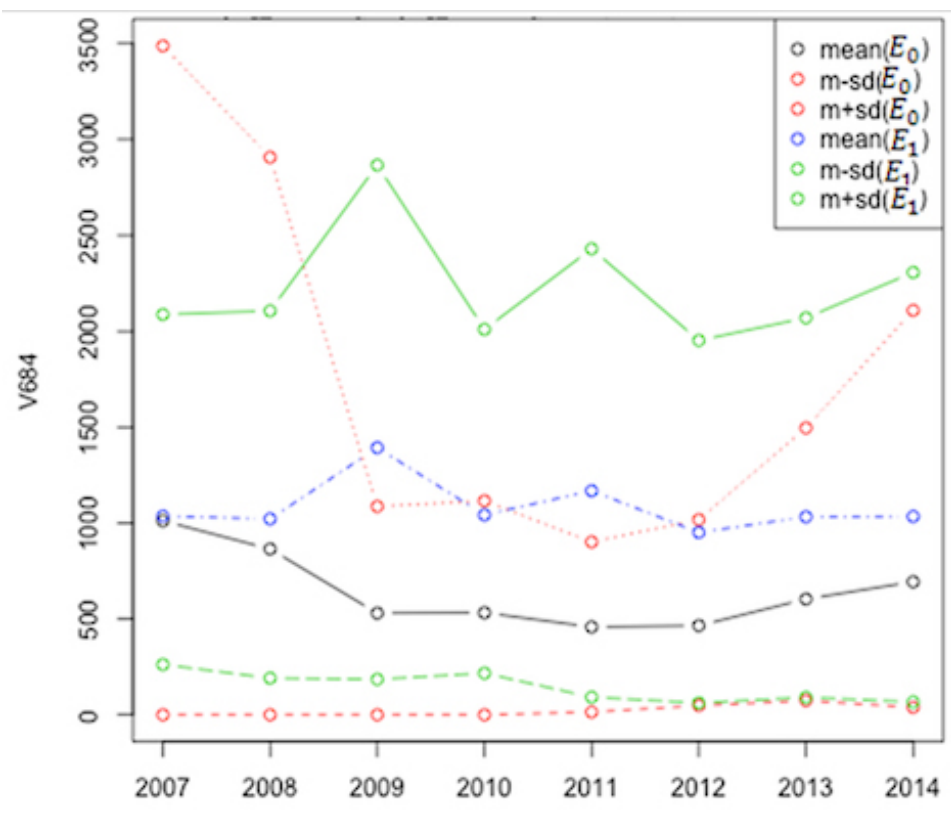

FIGURA 5

Comportamiento de los grupos de eficiencia

Fuente: elaboración propia.

\section{Discusión}

El presente estudio se destaca por ser pionero en la utilización del método MEA en Colombia, a efectos de fortalecer la investigación nacional de tipo exploratorio, específicamente en la medición del nivel de eficiencia sobre los servicios de $\mathrm{GO}, \mathrm{O}$ y $\mathrm{U}$ que prestan los hospitales públicos de nivel 1 de atención en el departamento del Valle del Cauca. A su vez, esta investigación tiene especial importancia, debido a que son los hospitales públicos de primer nivel con los que la población pobre asegurada (afiliados al régimen subsidiado) ${ }^{6}$ y no asegurada del Valle del Cauca tiene mayor contacto en lo que a servicios de salud se refiere.

La atención oportuna y pertinente en el servicio de $U$ constituye una base primordial en el buen desarrollo de una sociedad preparada ante eventualidades de carácter individual o grupal. La atención con calidad y eficiencia en el servicio GO es fundamental en el presente y en el futuro de un país, reflejado en el valor y el respeto a las mujeres como fuente de vida. Es de notar que aun cuando las enfermedades orales no conducen de manera inmediata a la muerte, como puede acontecer con los otros dos servicios considerados, dificultades en la salud bucal generalmente conllevan un intenso y prolongado dolor que incide sobre el comportamiento de las personas y su relación con el otro, afectando cualquiera de sus entornos (familiar, social o laboral). Por esta razón, la atención especializada en el servicio de O merece una visión destacada en la calidad de vida de cualquier ciudadano, y por tanto este servicio es también incluido como parte esencial de nuestro análisis.

Cada año, por normatividad, en Colombia (artículo 80, Ley 1438 de 2011) las direcciones departamentales, municipales y distritales son notificadas por el Ministerio de Salud y Protección Social 
acerca de la categorización del riesgo de los hospitales públicos, a partir de sus indicadores financieros y teniendo en cuenta sus condiciones de mercado, de equilibrio y viabilidad financiera. En la tabla 4 se presenta cómo los hospitales públicos de nivel 1 fueron categorizados por su riesgo, para el periodo comprendido entre el año 2012 hasta el 2016 inclusive. Como se puede apreciar, un porcentaje considerable (27.3\%, $52.3 \%, 43.2 \%, 36.4 \%$ y $50 \%$ ) de los hospitales fueron categorizados en nivel de riesgo o en peligro de quiebra (vigencias: 2012,2013,2014,2015 y 2016, respectivamente), situación que puede estar asociada a una gestión ineficiente de los recursos hospitalarios y a otros aspectos como la estructura de mercado, lo cual puede colocar en riesgo la calidad en la prestación de los servicios a la población pobre asegurada (44.55\%) y a la población pobre no asegurada (8.08\%) del departamento del Valle del Cauca. Adicionalmente, 34 de los 44 hospitales de nivel 1 se constituyen en la única entidad hospitalaria pública existente en el municipio a la que puede acceder la población.

TABLA 4

Categorización del riesgo de hospitales públicos de nivel 1

\begin{tabular}{|c|c|c|c|c|c|}
\hline RIESGO & $\begin{array}{c}\mathbf{2 0 1 2} \\
\text { (Resolución } \\
\text { 2509) }\end{array}$ & $\begin{array}{c}\mathbf{2 0 1 3} \\
\text { (Resolución } \\
\mathbf{1 8 7 7}\end{array}$ & $\begin{array}{c}\mathbf{2 0 1 4} \\
\text { (Resolución } \\
\text { 2090) }\end{array}$ & $\begin{array}{c}\mathbf{2 0 1 5} \\
\text { (Resolución } \\
\text { 1893) }\end{array}$ & $\begin{array}{c}\mathbf{2 0 1 6} \\
\text { (Resolución } \\
\text { 2184) }\end{array}$ \\
\hline ALTO & 2 & 6 & 7 & 1 & 2 \\
\hline MEDIO & 3 & 7 & 7 & 3 & 8 \\
\hline BAJO & 7 & 10 & 5 & 12 & 12 \\
\hline SIN & 32 & 21 & 25 & 28 & 22 \\
\hline TOTAL & 44 & 44 & 44 & 44 & 44 \\
\hline
\end{tabular}

Fuente: Ministerio de Salud y Protección Social, 2016.

A pesar de que nuestros resultados muestran que durante el periodo de estudio (2007-2014), los hospitales públicos, en su mayoría, mantuvieron un bajo nivel de eficiencia en la prestación de los tres servicios, encontramos en la tabla 2 que en los años 2011, 2013 y 2014 más del 50\% de los hospitales fueron eficientes en U. En efecto, aunque en el año 2010 el nivel en U fue el más bajo, hasta el punto de casi ser alcanzado en nivel por GO, el nivel de eficiencia de $\mathrm{U}$ es muy superior al de los otros dos servicios, durante todo el periodo de estudio. Por otro lado, si bien GO consiguió mejorar su eficiencia en el año 2010, prontamente el nivel de eficiencia adquirido disminuyó en los años posteriores. Por su parte, $\mathrm{O}$ pareciera tener un comportamiento inversamente proporcional al de GO. En términos generales, todo refleja que la estrategia seguida por los tres servicios de salud, especialmente por $\mathrm{GO}$ y $\mathrm{O}$, no es la mejor y necesita ser readaptada lo más pronto posible.

En los datos que se presentan en la tabla 3, en el año 2009 se destacan dos situaciones: la primera, el año con el mayor porcentaje en promedio, 39.3\%, en el que las veces que en los tres servicios de salud utilizaron los insumos, lo hicieron de manera ineficiente. La segunda, la variable de entrada con el indicador de ineficiencia más alto corresponde a V258 en el servicio de GO, con un valor de 0.75 . Esto significa que el $75 \%$ de las veces fue utilizado de forma no eficiente, lo cual es un porcentaje muy alto, que llama la atención a una revisión más rigurosa sobre la forma en que se suministran los insumos hospitalarios. Caso contrario sucedió con los insumos V208 y V679, que tuvieron una mejor utilización en términos de eficiencia, tanto en el servicio de U como en GO y O, en el año 2014.

Como era de esperarse, por los resultados obtenidos en las ratios de eficiencia en el servicio $\mathrm{U}$, se obtienen indicadores de ineficiencia en menor proporción para este servicio. En la figura 2 podemos apreciar que el 2009 representa el año en que los insumos fueron usados de manera más ineficiente, siendo en este mismo cuando los hospitales públicos lograron su más bajo porcentaje de eficiencia (23\%) durante el periodo de estudio (véase tabla 2). En la figura 3 podemos observar cómo entre el año 2010 y el 2011 se reduce en un alto porcentaje el uso ineficiente de los insumos en $\mathrm{O}$. 
Respecto al servicio de salud de U, como se señaló, encontramos que sus variables de entrada tuvieron el más bajo porcentaje de utilización ineficiente con respecto a los demás insumos de GO y O. Cabe destacar que la variable V208 en U fue la que obtuvo los menores índices de ineficiencia durante el periodo de estudio. Caso contrario sucedió con la variable V258 en GO, la cual mostró que fue usada de manera ineficiente todas las veces en las unidades hospitalarias.

En términos generales, podemos resaltar que los hospitales públicos lograron una mejor utilización de los insumos V208, V254, V256, V257 y V679 en U. Sin embargo, estos presentaron en términos relativos un exceso de entradas en GO durante todo el periodo de estudio, situación que puede estar correlacionada con el hecho de que la tasa bruta de natalidad en el Valle del Cauca presentó una disminución de manera gradual en el tiempo, con un valor máximo de 26.41 por mil habitantes entre 1985 y 1990, mientras que en el periodo 2010-2015 fue de 16.44. Esto significa una reducción del 37\% en la tasa de natalidad, según datos de la Secretaría Departamental de Salud [28].

Como se puede ver en la figura 5 , en cada año existen grandes diferencias en el comportamiento de la variable entre los dos grupos. Cabe anotar que existen hospitales cuyo nivel de eficiencia en los servicios de $\mathrm{GO}$ y de $\mathrm{O}$ no se encuentra en ninguno de los dos grupos. Esto significa que presentan un nivel de eficiencia superior al de los hospitales menos eficientes $E_{0}$ pero a la vez menor que el de los hospitales más eficientes $E_{0}$. Lo contrario ocurre en el servicio U, donde la totalidad de los hospitales se divide entre los dos grupos. Resaltamos que en el servicio de $U$ tanto los hospitales públicos más eficientes como los menos eficientes hicieron un mejor uso de la variable de entrada V254 en los años 2013 y 2014, y de la variable de salida V684 en el año 2007.

Como principal limitación de este estudio cabe señalar la calidad de la información contenida en la base de datos SIHO, debido a que los insumos/inputs y outputs/productos no están discriminados según el tipo o área de servicios de salud que los hospitales públicos ofertan a la población, situación que generó una dificultad al momento de organizar los datos, como la imposibilidad de incluir el recurso humano que presta sus servicios en las áreas de GO, O y U. A su vez, este módulo carece de información sobre el personal médico, asistencial y administrativo que se vincula mediante un contrato de prestación de servicios.

En Colombia hasta el momento no se dispone de investigaciones que aborden la medición de la eficiencia sobre servicios de salud GO, O y U que prestan los hospitales públicos de nivel 1 de atención, mediante la utilización de un método como el MEA, tal vez debido a las dificultades en la obtención de información suficiente y de calidad, o también porque a nivel internacional son numerosos los estudios sobre la evaluación de la eficiencia hospitalaria que usan el método DEA [29,30]. De todas formas, encontramos que este estudio, con respecto a otros $[12,18,19,20,21,22,23,24,25]$, aporta información más específica, como que durante el periodo 2007-2014 todos los hospitales públicos fueron poco eficientes en la prestación de servicios de GO, O y U; gráficos que representan las diferencias entre los hospitales más y menos eficientes, a la vez que consigue determinar de manera detallada en qué año y cuál insumo fue usado de manera ineficiente. Con datos como los anteriores, las autoridades ejecutivas locales y los gerentes de los hospitales tendrán a su disposición mejor información al momento de la toma de decisiones.

\section{Conclusiones}

La utilización del método MEA centrado en tres indicadores: ratios de eficiencia, índices de ineficiencia y cálculo del $\mathrm{NC}$ valor, nos muestra que los hospitales públicos tuvieron en términos relativos un desempeño poco eficiente en la utilización de los inputs empleados y en los outputs conseguidos en cada uno de los servicios de GO, O y U prestados durante el periodo 2007-2014.

En 2014, el 56.8\% (25 de 44) de los hospitales públicos fueron categorizados sin nivel de riesgo financiero (véase tabla 4), sin embargo, en ese mismo año los ratios de eficiencia obtenidos por el modelo MEA 
determinaron que el 15.9\% (7 de 44), el 27.3\% (12 de 44) y el 54.5\% (24 de 44) de los hospitales públicos fueron eficientes en la prestación de los servicios $\mathrm{GO}, \mathrm{O}$ y $\mathrm{U}$, respectivamente. En síntesis, reducir el peligro de una quiebra financiera de una institución hospitalaria necesariamente no significa que los recursos físicos o insumos se han asignado de manera eficiente en la prestación de los servicios de salud a las personas pobres aseguradas y no aseguradas.

Si se compara los tres servicios estudiados, los resultados reflejan un nivel superior de eficiencia en $U$ en la mayoría de los hospitales vallecaucanos. Sin embargo, durante el periodo de estudio, el servicio U alcanzó valores-NC mucho mayores para todas las variables que los valores- $\mathrm{NC}$ obtenidos en los servicios $\mathrm{GO}$ y $\mathrm{O}$ en sus correspondientes variables. Esto implica que los hospitales menos eficientes en el servicio de $\mathrm{U}$ necesitan considerar cambios más grandes en su estructura y funcionalidad que les permitan tener un mejor aprovechamiento de los insumos. Adicionalmente, el grupo de hospitales más eficientes obtuvo un mayor desempeño, tanto de las variables de entrada como de las de salida, que el grupo de hospitales menos eficientes en la prestación de los tres servicios.

Respecto a los indicadores de ineficiencia, la situación puede considerarse crítica, dado que en promedio el 40\% de veces en que fueron usados las variables de entrada en $\mathrm{GO}$ y O, lo hicieron de manera ineficiente. Consideramos que la desacertada gestión de los recursos en los hospitales pueda obedecer, a saber: a la falta de una estructura legal de incentivos económicos basados en la productividad del gerente del hospital y su equipo de trabajo; a una conducta de riesgo moral de las entidades que ejercen seguimiento y control; a unas reglas no formales que motivan a los servidores públicos a asumir un comportamiento deshonesto; a la demora en los pagos que las empresas promotoras de salud (EPS) deben hacer a los hospitales públicos por los servicios prestados a sus afiliados, entre otras múltiples razones. Entonces, esperamos que los tomadores de decisiones con los resultados obtenidos en este estudio puedan reducir los problemas de información asimétrica, de tal manera que establezcan estrategias de monitoreo, seguimiento y control a la forma en que el personal médicoasistencial hace uso de los insumos que tienen a disposición para la prestación de los servicios de salud de $\mathrm{GO}, \mathrm{O}$ y U.

Para terminar, invitamos a políticos, autoridades locales, gerentes de hospitales y a instituciones de vigilancia y control a que utilicen este tipo de métodos o herramientas técnicas, a fin de establecer un mejor sistema de información para el seguimiento, el monitoreo, el control y la evaluación a las actividades que llevan a cabo los hospitales públicos.

\section{Agradecimientos}

Proyecto de investigación derivado del proyecto Medición de la eficiencia en los hospitales públicos del departamento del Valle del Cauca durante el periodo 2010-2014. Inicio: 1.o de noviembre de 2016; finalización: 1.o de julio de 2018. Investigación financiada parcialmente con recursos portugueses a través del Centro de Investigación y Desarrollo en Matemáticas y Aplicaciones (CIDMA) de la Universidade de Aveiro (Portugal), y la Fundación Portuguesa para la Ciencia y la Tecnología (FCT), dentro del proyecto con referencia UID/MAT/04106/2013 financiado por el Proyecto 3599 - Promover un Fondo de Desarrollo Económico y Financiero (3599-PPCDT) y FEDER a través de COMPETE 2020, Programa Operacional Competividad e Internacionalización (POCI), y por fondos nacionales a través de FCT. Murillo también recibe el apoyo de la beca FCT post-doc con referencia SFRH/BPD/97085/2013. Asimismo, la investigación cuenta con recursos de la Dirección General de Investigaciones de la Universidad Santiago de Cali (Colombia), Código institucional de aprobación: DGI-COCEIN-No.311-621116-D45. 


\section{Referencias}

1. Mushkin SJ. Health as an investment. Part 2: Investment in human beings. Journal of Political Economy. 1962; 70(5):129-57.

2. Grossman M. On the concept of health capital and the demand for health. The Journal of Political Economy, 1972; $80(2): 223-55$.

3. Barro RJ. Determinants of economic growth: a cross-country empirical study. NBER Working Paper No. 5698, NBER Program(s): EFG, Issued in 1996.

4. Infante A, Mata I, López-Acuna D. Reforma de los sistemas de salud en America Latina y el Caribe: situacion y tendencias. Rev Panam Salud Publica 2000; 8(1/2).

5. Tono RT. Los hospitales colombianos en el contexto de Latinoamérica y el Caribe. Documento de Trabajo N.o 8; Bogotá: Fundación Corona y Fundación Ford, 2002.

6. Bogetoft P, Otto L. Benchmarking with DEA, SFA, and R. Nueva York: Springer, Science \& Business Media; 2011.

7. Hougaard JL, Overgaard C. Frontier estimation methods as management tools in health care production: a case of staff utilization in Danish orthopaedic surgery units. Nationaløkonomisk tidsskrift, 2006; 144(3):377-92.

8. Hougaard JL, Kronborg D, Overgård, C. Improvement potential in Danish elderly care. Health Care Management Science, 2004; 7(3):225-35.

9. Asmild M, Matthews K. Multi-directional efficiency analysis of efficiency patterns in Chinese banks 1997-2008. European Journal of Operational Research, 2012; 219:434-41.

10. Wang K, Wei YM, Zhang X. Energy and emissions efficiency patterns of Chinese regions: A multi-directional efficiency analysis. Applied Energy, 2013; 104:105-16.

11. Asmild M, Bale ${ }^{\vee}$ zentis T, Hougaard JL. Multi-directional program efficiency: The case of Lithuanian family farms. Journal of Productivity Analysis, 2016; 45:23-33.

12. Giedion U, Morales L, Muriel L, Rodríguez CJ. Medición de la eficiencia económica y de gestión en los hospitales públicos del Distrito Capital. Secretaría Distrital de Salud, Bogotá (Colombia), 1999.

13. Puig-Junoy J. Eficiencia en la Atención Primaria de Salud: una revisión crítica de medidas de frontera. Rev Esp Salud Pública, 2000; 74(5-6):483-95.

14. Asmild M, Baležentis T, Hougaard JL. Multi-directional Productivity Change: MEA Malmquist, Journal of Productivity Analysis, 2016; 46:109-19.

15. Asmild M, Holvad T, Hougaard J, Kronborg D. Railway reforms: do they inuence operating efficiency? Transportation, 2009; 36(5):617-38.

16. Gongbing B, Pingchun W, Feng Y, Liang L, Energy and environmental efficiency of China's transportation sector: a multidirectional analysis approach. Mathematical Problems in Engineering, 2014; 1-12. http://dx.doi.org/10 $.1155 / 2014 / 539596$

17. Wang K, Yu S, Li MJ, Wei Y. Multi-directional efficiency analysis-based regional industrial environmental performance evaluation of China. Natural Hazards Supplement, 2015; 75(2): 273-99. Disponible en: https:// doi.org/10.1007/s11069-014-1097-4

18. Rojas M. Evaluación de la eficiencia en las empresas sociales del Estado del departamento del Tolima: el caso de los hospitales de mediana complejidad [tesis de maestría]. Bogotá: Universidad de los Andes; 2009.

19. Mora AH, Morales LG. Consideraciones sobre la evaluación de la eficiencia relativa de los hospitales colombianos. Revista Universitas Económica, 1997 junio, IX (3).

20. Polanía C. Una aplicación de Data Envelopment Analysis: análisis comparativo de eficiencia entre hospitales en Colombia [tesis de maestría]. Bogotá: Universidad de los Andes; 1999.

21. Nupia OA, Sánchez F. Eficiencia de los hospitales públicos de Bogotá. Revista Desarrollo y Sociedad, 2001; (48):101-36.

22. Pinzón M. Medición de eficiencia técnica relativa en hospitales públicos de baja complejidad mediante la metodología Data Envelopment Analysis (DEA). Bogotá: DNP, Archivos de Economía, documento 245; 2003. 
23. Sarmiento A, Castellanos W, Nieto A, Alonso C, Pérez C. Análisis de eficiencia técnica de la red pública de prestadores de servicios de salud dentro del Sistema General de Seguridad Social en Salud. Revista Gerencia y Políticas de Salud. 2006; 5(11).

24. Toro G, Erotida E, Mutis G. Medición de la eficiencia en las instituciones prestadoras de servicio de salud públicas mediante técnicas multivariadas y análisis envolvente de datos. Bogotá: Universidad de los Andes; 2006.

25. Fontalvo TJ, De la Hoz G. Eficiencia de los hospitales de Bolívar-Colombia, por medio de análisis envolvente de datos. Dimensión Empresarial, 2016; 14(1): 95-108. Disponible en: https://doi.org/10.15665/rde.v14i1.469

26. Bogetdet P, Hougaard JL. Efficiency evaluations based on potential (non-proportional) improvements, Journal de Productivity Analysis, 1999; 12(3): 233-47.

27. Inman HF, Bradley Jr. EL. The over-lapping coefficient as a measure de agreement between probability distributions and point estimation de the overlap de two normal densities, Communications in Statistics, Theory and Methods, 1989; 18(10): 3851-74.

28. Secretaría Departamental de Salud del Valle del Cauca (SDSV). Análisis de situación de salud con el modelo de los determinantes sociales Valle del Cauca 2015. Cali: SDSV; 2016.

29. Herrero TL, Martín JJ, López del Amo GM. Eficiencia técnica de los hospitales públicos y de las empresas públicas hospitalarias de Andalucía. Gac Sanit 2015; 29(4):274-81. Disponible en: https://doi.org/10.1016/j.gaceta.20 15.03.001

30. Pérez C, Ortega MI, Ocaña R, Martín JJ. Análisis de la eficiencia técnica en los hospitales del Sistema Nacional de Salud español. Gac Sanit 2017; 31(2):108-15. Disponible en: https://doi.org/10.1016/j.gaceta.2016.10.007 


\section{Apéndices}

TABLA 5

DATOS ESTADÍSTICOS DEL SERVICIO DE SALUD U

\begin{tabular}{|c|c|c|c|c|c|c|c|c|}
\hline $\mathbf{v}$ & 2007 & 2008 & 2009 & 2010 & 2011 & 2012 & 2013 & 2014 \\
\hline \multirow{3}{*}{ V208 } & 936674 & 967509 & 1131975 & 1081613 & 1044677 & 1009634 & 1058041 & 1060662 \\
\hline & $20 \$ 14.97$ & 21500.2 & 25155 & 24035.84 & 23215.84 & 22436.31 & 23512.02 & 23570.26 \\
\hline & 26478.18 & 26248.26 & 31970.22 & 31531.46 & 31267.55 & 28529.41 & 28819.53 & 27868.89 \\
\hline \multirow{3}{*}{ V253 } & 589 & 611 & 630 & 629 & 632 & 620 & 673 & 676 \\
\hline & 13.08 & 13.57 & 14 & 13.97 & 14.04 & 13.77 & 14.95 & 15.02 \\
\hline & 6 & 6.15 & 6.03 & 6.01 & 6.57 & 5.81 & 11343 & 11.37 \\
\hline \multirow{3}{*}{ V254 } & 176 & 208 & 209 & 223 & 246 & 289 & 272 & 277 \\
\hline & 3.91 & 4.62 & 4.64 & 4.95 & 5.46 & 6.42 & 6.04 & 6.15 \\
\hline & 3.57 & 3.89 & 3.87 & 3.71 & 4.84 & 6.12 & 5.35 & 5.35 \\
\hline \multirow{3}{*}{ V256 } & 83 & 89 & 95 & 96 & 96 & 102 & 105 & 110 \\
\hline & 1.84 & 1.97 & 2.11 & 2.13 & 2.13 & 2.26 & 2.33 & 2.44 \\
\hline & 1.34 & 1.87 & 1.83 & 1.65 & 1.64 & 1.71 & 1.83 & 1.82 \\
\hline \multirow{3}{*}{ V257 } & 18 & 19 & 16 & 17 & 16 & 51 & 14 & 12 \\
\hline & 0.4 & 0.42 & 0.35 & 0.37 & 0.35 & 1.13 & 0.31 & 0.26 \\
\hline & 0.65 & 0.72 & 0.6 & 0.64 & 0.67 & 5.05 & 0.76 & 0.65 \\
\hline \multirow{3}{*}{ V258 } & 63 & 65 & 57 & 57 & 57 & 58 & 59 & 55 \\
\hline & 1.4 & 1.44 & 1.26 & 1.26 & 1.26 & 1.28 & 1.31 & 1.22 \\
\hline & 0.65 & 1.11 & 0.53 & 0.53 & 0.53 & 0.58 & 0.55 & 0.47 \\
\hline \multirow{3}{*}{ V679 } & 908286 & 876909 & 1072880 & 929367 & 784434 & 891645 & 916910 & 717720 \\
\hline & 20184.13 & 19486.86 & 23841.77 & 20652.6 & 17431.86 & 19814.33 & 20375.77 & 15949.33 \\
\hline & 25391.88 & 24095.79 & 30664.75 & 21019.94 & 14747.86 & 21593.45 & 26189.54 & 15232.34 \\
\hline \multirow{3}{*}{ V240 } & 2549470 & 2753029 & 2799522 & 3073644 & 3235639 & 3644587 & 4005134 & 4161462 \\
\hline & 56654.88 & 61178.42 & 62211.6 & 68303.2 & 71903.08 & 80990.82 & 89002.97 & 92476.93 \\
\hline & 87178.62 & 89907.21 & 93321.21 & 105657.8 & 111350.7 & 115445.9 & 128473.8 & 131107.8 \\
\hline \multirow{3}{*}{ V241 } & 264983 & 220571 & 238532 & 242849 & 267097 & 239630 & 259416 & 291264 \\
\hline & 5888.51 & 4901.57 & 5300.71 & 5396.64 & 5935.48 & 5325.11 & 5764.8 & 6472.53 \\
\hline & 17744.71 & 9225.83 & 9911.81 & 10693.45 & 9480.22 & 7211.01 & 7885.68 & 9862.65 \\
\hline \multirow{3}{*}{ V444 } & 56689 & 66502 & 52974 & 68172 & 69065 & 67982 & 67867 & 62473 \\
\hline & 1259.75 & 1477.82 & 1177.2 & 1514.93 & 1534.77 & 1510.71 & 1508.15 & 1388.28 \\
\hline & 1533.78 & 1382.66 & 969.53 & 1767.54 & 1357.62 & 1238.86 & 1526.3 & 1284.86 \\
\hline \multirow{3}{*}{ V684 } & 39745 & 37611 & 35817 & 34591 & 32506 & 29554 & 31954 & 32200 \\
\hline & 883.22 & 835.8 & 795.93 & 768.68 & 722.35 & 656.75 & 710.08 & 715.55 \\
\hline & 1685.66 & 1430.6 & 890.14 & $\$ 23.61$ & 830.1 & 691.97 & 819.33 & 1124.48 \\
\hline
\end{tabular}

Fuente: elaboración propia 
TABLA 6

DATOS ESTADÍSTICOS DEL SERVICIO DE SALUD O

\begin{tabular}{|c|c|c|c|c|c|c|c|c|}
\hline v & 2007 & 2008 & 2009 & 2010 & 2011 & 2012 & 2013 & 2014 \\
\hline \multirow{3}{*}{ V211 } & 347.803 & 319.529 & 341.114 & 337.279 & 327.707 & 368.355 & 404.356 & 423.503 \\
\hline & $7.728,95$ & $7.100,64$ & $7.580,31$ & $7.495,08$ & $7.282,37$ & $8.185,66$ & $8.985,68$ & $9.411,17$ \\
\hline & $9.327,28$ & $10.171,01$ & $11.011,3$ & $11.447,25$ & 11.627 & $12.758,09$ & $13.550,79$ & $14.372,53$ \\
\hline \multirow{3}{*}{ V259 } & 332 & 353 & 349 & 350 & 354 & 385 & 370 & 375 \\
\hline & 7,37 & 7,84 & 7,75 & 7,77 & 7,86 & 8,55 & 8,22 & 8,33 \\
\hline & 10,72 & 10,64 & 11,72 & 12,02 & 11,38 & 11,48 & 10,92 & 11,27 \\
\hline \multirow{3}{*}{ V681 } & 571.229 & 459.326 & 544.101 & 501.051 & 468.441 & 484.297 & 504.199 & 484.001 \\
\hline & $12.693,97$ & $10.207,24$ & $12.091,13$ & $11.134,46$ & $10.409,8$ & $10.762,15$ & $11.204,42$ & $10.755,57$ \\
\hline & $17.430,5$ & $11.121,29$ & $14.671,84$ & $13.230,8$ & $13.360,08$ & $13.300,94$ & $14.396,66$ & $15.180,13$ \\
\hline \multirow{3}{*}{ V212 } & 534.772 & 569.403 & 527.915 & 519.616 & 519.409 & 575.455 & 535.005 & 553.721 \\
\hline & $11.883,82$ & $12.653,4$ & $11.731,44$ & $11.547,02$ & $11.542,42$ & $12.787,88$ & 11.889 & $12.304,91$ \\
\hline & $15.796,83$ & $20.684,42$ & $19.985,32$ & $19.581,48$ & $19.774,66$ & $20.672,77$ & $19.894,34$ & $22.062,52$ \\
\hline \multirow{3}{*}{ V213 } & 664.128 & 720.023 & 793.443 & 825.601 & 821.052 & 900.641 & 964.360 & 955.078 \\
\hline & $14.758,4$ & $16.000,51$ & $17.632,06$ & $18.346,68$ & $18.245,6$ & $20.014,24$ & $21.430,22$ & $21.223,95$ \\
\hline & $25.626,26$ & $28.461,92$ & $29.307,41$ & $30.658,62$ & $29.526,09$ & $30.824,66$ & $33.952,84$ & $34.166,78$ \\
\hline \multirow{3}{*}{ V214 } & 90.163 & 91.308 & 97.532 & 102.836 & 11.7898 & 122.488 & 125.280 & 125.331 \\
\hline & $2.003,62$ & $2.029,06$ & $2.167,37$ & $2.285,24$ & $2.619,95$ & 2721,95 & 2.784 & $2.785,13$ \\
\hline & $2.864,74$ & $3.091,97$ & $3.166,36$ & $3.392,57$ & $6.621,52$ & $6.539,82$ & $6.747,35$ & $7.353,22$ \\
\hline
\end{tabular}

Fuente: elaboración propia.

TABLA 7

Datos estadísticos del servicio de salud GO

\begin{tabular}{|c|c|c|c|c|c|c|c|c|}
\hline $\mathbf{v}$ & 2007 & 2008 & 2009 & 2010 & 2011 & 2012 & 2013 & 2014 \\
\hline \multirow{3}{*}{ V205 } & 221523 & 196291 & 201269 & 228487 & 236163 & 243951 & 219967 & 214105 \\
\hline & 4922.73 & 4362.02 & 4472.64 & 5077.48 & 5248.06 & 5421.13 & 4888.15 & 4757.88 \\
\hline & 7809.11 & 6108.86 & 6578.46 & 8784.86 & 8788.93 & 9178.9 & 6999.62 & 6956.67 \\
\hline \multirow{3}{*}{ V253 } & 589 & 611 & 630 & 629 & 632 & 620 & 673 & 676 \\
\hline & 13.08 & 13.57 & 14 & 13.97 & 14.04 & 13.77 & 14.95 & 15.02 \\
\hline & 6 & 6.15 & 6.03 & 6.01 & 6.57 & 5.81 & 11.34 & 11.37 \\
\hline \multirow{3}{*}{ V258 } & 63 & 65 & 57 & 57 & 57 & 58 & 59 & 55 \\
\hline & 1.4 & 1.44 & 1.26 & 1.26 & 1.26 & 1.28 & 1.31 & 1.22 \\
\hline & 0.65 & 1.12 & 0.53 & 0.53 & 0.53 & 0.58 & 0.55 & 0.47 \\
\hline \multirow{3}{*}{ V220 } & 11798 & 11819 & 11124 & 9573 & 9178 & 7915 & 7990 & \& 191 \\
\hline & 262.17 & 262.64 & 247.2 & 212.73 & 203.95 & 175.88 & 177.55 & 182.02 \\
\hline & 467.09 & 479.33 & 449.43 & 398 & 372.34 & 330.29 & 325.63 & 351.7 \\
\hline \multirow{3}{*}{ V445 } & 7436 & 7495 & 8374 & 7156 & 6994 & 6335 & 5504 & 5971 \\
\hline & 165.24 & 166.55 & 186.08 & 159.02 & 155.42 & 140.77 & 122.31 & 132.68 \\
\hline & 249.13 & 282.18 & 332.59 & 248.86 & 199.13 & 192.72 & 149.25 & 302.81 \\
\hline
\end{tabular}

Fuente: elaboración propia

\section{Notas}

* Artículo de investigación

$1 \quad$ Ley 100 de 1993, Decreto 1876 de 1994, Ley 1122 del 2007 y Ley 1438 del 2011.

2 En Colombia se adopta el modelo piramidal clásico, el cual fue diseñado como sigue. Nivel I: consulta externa y hospitalización en medicina general; nivel II: consulta externa, cirugía general, ginecología y obstetricia, medicina interna, pediatría y especialidades quirúrgicas con requerimiento intermedio de hospitalización; nivel III: consulta externa, cirugía general, ginecología y obstetricia, medicina interna, pediatría y todas las especialidades y subespecialidades en el nivel avanzado de hospitalización. 
Otros artículos con aplicaciones en MEA se referencian con mayor detalle en la sección "DEA versus MEA".

4 El Hospital Geriátrico y Ancianato San Miguel de la Ciudad de Cali no fue considerado en nuestro estudio, debido a que su portafolio de servicios está dirigido solamente a la población de la tercera edad. A su vez, su infraestructura es diferente de la de los otros hospitales objeto de estudio.

5 Los municipios de Roldanillo, Sevilla y Zarzal no cuentan con hospitales públicos de nivel I de atención.

6 El régimen subsidiado es el mecanismo mediante el cual la población urbana y rural más pobre, sin capacidad de pago - madres durante el embarazo, parto, posparto y periodo de lactancia; madres comunitarias; mujeres cabeza de familia; niños menores de un año y menores en situación irregular - tendrá acceso a los servicios de salud a través de un subsidio a la demanda que otorga el Estado colombiano (numeral 2, artículo 157, Ley 100 de 1993).

Financiamiento de la investigación

Fuente: Centro de Investigación y Desarrollo en Matemáticas y Aplicaciones (CIDMA) de la Universidade de Aveiro (Portugal), y la Fundación Portuguesa para la Ciencia y la Tecnología (FCT)

No de contrato: UID/MAT/04106/2013

Beneficiario: Análisis de eficiencia multidireccional en hospitales públicos del Valle del Cauca

Fuente: Proyecto 3599 - Promover un Fondo de Desarrollo Económico y Financiero y FEDER No de contrato: 3599-PPCDT

Fuente: FCT

No de contrato: SFRH/BPD/97085/2013

Beneficiario: Kelly Patricia Murillo

Fuente: Universidad Santiago de Cali (Colombia)

No de contrato: DGI-COCEIN-No.311-621116-D45

\section{Licencia Creative Commons CC BY 4.0}

Como citar este artículo: Murillo KP, Rocha EAM, García González CA. Análisis de eficiencia multidireccional en hospitales públicos del Valle del Cauca. Rev Gerenc Polít Salud. 2017; 17(34): 1-20. https://doi.org/10.11144/Javeriana.rgps17-34.aemh 